\title{
Deep learning-driven catheter tracking from bi-plane $X$-ray fluoroscopy of 3D printed heart phantoms
}

\author{
Matin Torabinia ${ }^{1,2}$, Alexandre Caprio ${ }^{1,2}$, Sun-Joo Jang ${ }^{1,2}$, Tianyu Ma ${ }^{2,3}$, Honson Tran ${ }^{1,2}$, Lina Mekki ${ }^{1,2}$, Isabella \\ Chen $^{1,2}$, Mert Sabuncu ${ }^{2,3}$, S. Chiu Wong ${ }^{4}$, Bobak Mosadegh ${ }^{1,2}$ \\ 'Dalio Institute of Cardiovascular Imaging, NewYork-Presbyterian Hospital and Weill Cornell Medicine, New York, NY 10021, \\ USA. \\ 2Department of Radiology, Weill Cornell Medicine, New York, NY 10021, USA. \\ ${ }^{3}$ School of Electrical and Computer Engineering, Cornell Univesity, Ithaca, NY 10021, USA \\ ${ }^{4}$ Division of Cardiology, Department of Medicine, Weill Cornell Medicine, New York, NY 10021, USA
}

Correspondence to: Dr. Bobak Mosadegh, Dalio Institute of Cardiovascular Imaging, Department of Radiology, NewYorkPresbyterian Hospital and Weill Cornell Medicine, 1196 York Avenue, Bronk 908B, New York, NY 10065, USA.

E-mail: bom2008@med.cornell.edu

\begin{abstract}
How to cite this article: Torabinia M, Caprio A, Jang SJ, Ma T, Tran H, Mekki L, Chen I, Sabuncu M, Wong SC, Mosadegh B. Deep learning-driven catheter tracking from bi-plane X-ray fluoroscopy of 3D printed heart phantoms. Mini-invasive Surg 2021;5:32. https://dx.doi.org/10.20517/2574-1225.2021.63
\end{abstract}

Received: 8 May 2021 First Decision: 25 May 2021 Revised: 27 May 2021 Accepted: 7 Jun 2021 First online: 9 Jun 2021

Academic Editors: Bobak Mosadegh, Giulio Belli Copy Editor: Xi-Jun Chen Production Editor: Xi-Jun Chen

\begin{abstract}
Minimally invasive surgery (MIS) has changed not only the performance of specific operations but also the more effective strategic approach to all surgeries. Expansion of MIS to more complex surgeries demands further development of new technologies, including robotic surgical systems, navigation, guidance, visualizations, dexterity enhancement, and 3D printing technology. In the cardiovascular domain, 3D printed modeling can play a crucial role in providing improved visualization of the anatomical details and guide precision operations as well as functional evaluation of various congenital and congestive heart conditions. In this work, we propose a novel deep learning-driven tracking method for providing quantitative 3D tracking of mock cardiac interventions on customdesigned 3D printed heart phantoms. In this study, the position of the tip of a catheter is tracked from bi-plane fluoroscopic images. The continuous positioning of the catheter relative to the 3D printed model was co-registered in a single coordinate system using external fiducial markers embedded into the model. Our proposed method has the potential to provide quantitative analysis for training exercises of percutaneous procedures guided by bi-plane fluoroscopy.
\end{abstract}


Keywords: Catheter tracking, image guidance, deep learning, 3D printing, minimally invasive surgery, 3D trajectory, percutaneous interventions, patient-specific

\section{INTRODUCTION}

Since minimally invasive surgery (MIS) emerged in the 1980s, surgical skills and minimally invasive equipment have achieved significant advancements ${ }^{[1-3]}$. The minimally invasive approach holds a unique place for various surgical specialties, such as general surgery, urology $y^{[4]}$, thoracic surgery ${ }^{[5]}$, plastic surgery $y^{[6]}$, and cardiac surgery ${ }^{[7]}$. MIS has not only improved the recovery time of patient's from specific procedures, but is also enabled to provide improved outcomes ${ }^{[8,9]}$. These benefits to patients, hospitals and physicians have attributed to the rapid development of new MIS procedures, including cardiovascular diseases. The success of cardiac interventions over the last three decades has significantly reduce the mortality and morbidity of coronary, valvular, and various congenital diseases ${ }^{[1,11]}$. However, expansion of MIS to more complex surgeries demand further development of new technologies, including robotic surgical systems ${ }^{[12]}$, navigation ${ }^{[13]}$, guidance ${ }^{[14]}$, and visualizations ${ }^{[15]}$, dexterity enhancement ${ }^{[16]}$, and $3 \mathrm{D}$ printing technology $\mathrm{y}^{[17]}$.

In recent years, 3D printing technology has been attractive in diverse areas of medicine, including cardiovascular disease ${ }^{[18]}$. Increasing interest in anatomical modeling and the growing need for pre-operative planning using personalized anatomical models to test for device fit and practicing catheter positioning have encouraged the creation and evolution of 3D printed patient-specific model $s^{[19]}$. Recently, there are several studies showing various implementations of $3 \mathrm{D}$ printed heart models for different stages of structural heart interventions, such as pre-operative planning ${ }^{[20-23]}$, intra-operative models for enhanced structural orientation ${ }^{[24-26]}$, and evaluations of novel procedural pathways ${ }^{[27,28]}$. Garekar et al. ${ }^{[29]}$ utilized a 3D printed model for a double outlet right ventricle. The study showed the $3 \mathrm{D}$ printed model provided better intuition to decide on an operative approach than conventional imaging (i.e., echocardiography ${ }^{[29]}$. Chaowu et al ${ }^{[23]}$ demonstrated a $3 \mathrm{D}$ printed model for transcatheter closure of secundum atrial septal defect, where their findings suggested that $3 \mathrm{D}$ printing has the potential to screen for appropriate candidates. Other examples include tetralogy of Fallot ${ }^{[22,30]}$, hypoplastic left heart syndrome ${ }^{[31,32]}$, and ventricular septal defect ${ }^{[3,3,3]}$. Despite the successful implementation from prior work, the existing surgical planning from $3 \mathrm{D}$ printed models does not have methods to analyze how a catheter had actually maneuvered in the $3 \mathrm{D}$ printed model.

Our group recently reported a novel training system that provides catheter navigation in mixed reality (MR), with real-time visual feedback of a physical catheter's position within a patient-specific 3D heart model $^{[35]}$. This method used electromagnetic (EM) sensors to track the catheter position. Although this method is advantageous for portability, it has a low accuracy (up to $\sim 5 \mathrm{~mm}$ ), requires manual integration of sensors into a catheter, and the hardware not readily available in catheterization labs.

To address these limitations, we propose a novel deep learning-driven method for tracking a catheter in a $3 \mathrm{D}$ printed model from bi-plane fluoroscopic images acquired during the procedure. The catheter and heart position are co-registered in a single coordinate system using affine transformations based on four fiducial radiopaque markers, which are located on the $3 \mathrm{D}$ printed model. Additionally, the $3 \mathrm{D}$ trajectory of the catheter is produced, visualizing the path taken during the mock procedures. Our proposed method has the potential to provide quantitative analysis for training exercises of percutaneous procedures guided by biplane fluoroscopy. 


\section{Methodology}

A schematic of the proposed training system is shown in Figure 1, where a physician conducts a mock catheterization procedure using a bi-plane C-arm X-ray fluoroscopy machine on a patient-specific 3D printed model. The proposed image tracking aims to detect and co-register the catheter's $3 \mathrm{D}$ position and provide a 3D trajectory as quantitative feedback. Different features that are utilized for our proposed tracking system are described in detail in the following subsections, which are in the order by which this process is conducted.

\section{D printed phantom model}

To 3D print a patient-specific model, we used a 3D image processing software (Materialize Mimics Research software 21.0) to import an end-diastolic cardiac computed tomography (CT) scan as a DICOM (Digital Imaging Communication in Medicine) data file, shown in Figure 2A. In Mimics, the specific thresholds are set to segment the heart and the spine, enabling a $3 \mathrm{D}$ representation of the heart and spine in one mask while maintaining all the relative positions. Then, the $3 \mathrm{D}$ segmentation is saved as a STL file. To trim all the vessels, ribs, and other elements that are not necessary for the model, we used Geomagic Wrap (3D Systems Geomagic Corporation, NC, USA). Additionally, as depicted in Figure $2 \mathrm{~B}$ and $\mathrm{C}$, the artifacts were removed, and the meshwork was smoothed. Finally, using the "Shell" tool in Geomagic, the model obtained a water-tight thickness, and cleaned reconstructed objects were saved as STL files. Moreover, we utilized Solidworks software 2018 (Dassault Systems) to incorporate the supporting base structure for the heart and spine, fixing their relative distance during printing and use [Figure 2D and E]. This study used Stratasys Object Connex 260 printing system and the rigid and translucent material named VeroClear [Figure 2F]. Additionally, the post-printing process (i.e., removing supporting SUP705 Stratasys material) was conducted using a high-flow water jet cleaner (i.e., Powerblast) and art supply sculpting tools. In order to conduct mock catheterization procedures under a C-arm X-ray fluoroscopy machine, we integrated the phantom model into a 5-sided acrylic box (shoppopdisplays.com). The model is then glued in the center of the box with its inlet- and outlet-facing holes that were drilled at two opposite ends of the box [Figure $2 \mathrm{G}$ ]. Throughout the fluoroscopic imaging, the box is filled with water, eliminating artifacts from the $3 \mathrm{D}$ printed model.

\section{Deep learning architecture}

The advancement of deep learning architectures like convolutional neural networks (CNN) and deep autoencoders not only transformed typical computer vision tasks like object detection ${ }^{[36]}$, but are also efficient in other related tasks like classification ${ }^{[37]}$, localization ${ }^{[38]}$, tracking ${ }^{[39]}$, and image segmentation ${ }^{[40,41]}$. Ronneberger et al. ${ }^{[4]}$ proposed the state-of-the-art U-Net by replacing the pooling operators in Fully Convolutional Network ${ }^{[42]}$ with upsampling operators, allowing the input image's resolution retention. UNet's performance in segmenting medical images, notably with a small training dataset, promises the potential of such Encoder-Decoder architecture. The U-Net model was later extended for processing other medical images, including, but not limited to, the Xenopus kidney ${ }^{[43]}$ and MRI volume segmentation of prostate $^{[44]}$, retinal vessels, liver and tumors in CT scans, ischemic stroke lesion, intervertebral disc and pancreas ${ }^{[45-52]}$. In this work, to track the catheter's position from the bi-plane fluoroscopic images, we primarily leveraged the U-Net model to detect a radiopaque marker at the tip of the catheter. The details of implementation and framework will be discussed in the following sections.

\section{Collection and preparation of datasets}

All fluoroscopic images for training the deep learning U-Net model were acquired during the mock procedures in the catheterization lab at New York-Presbyterian Hospital. The datasets comprise 300 paired bi-plane images pertaining to the maneuvering of a catheter (OSCAR Deflectable Steerable Guiding Sheath, Destino $^{\text {mi }}$ Twist) within the patient-specific $3 \mathrm{D}$ printed model. The datasets were divided into 3 parts: (1) 

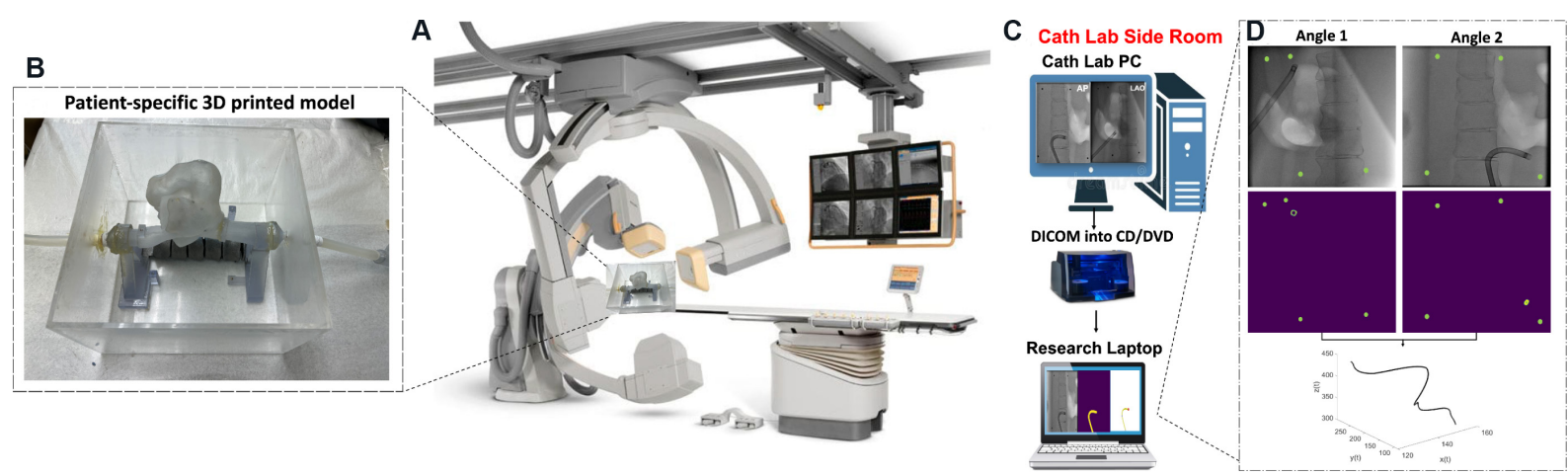

Figure 1. Schematic of the proposed training system. (A) Image of 3D printed heart model on a bi-plane C-arm. (B) Magnified view of patient-specific 3D printed heart model. (C) Schematic of image transfer process and post-processed catheter tracking. (D) Imageprocessing and deep learning steps of bi-plane images with tracking plot.

A DICOM File in Mimics
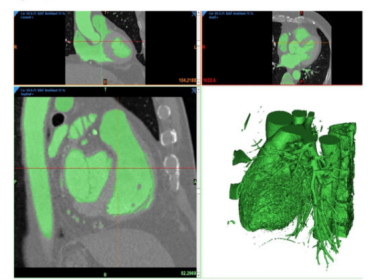

D Import STL in Solidworks

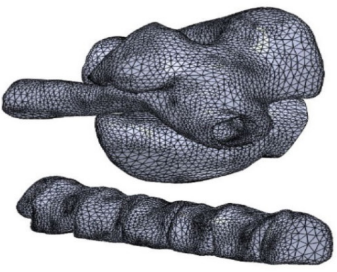

B Geomagic Wrap

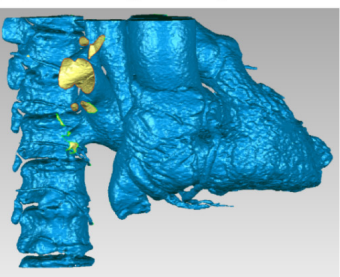

E supporting base structure

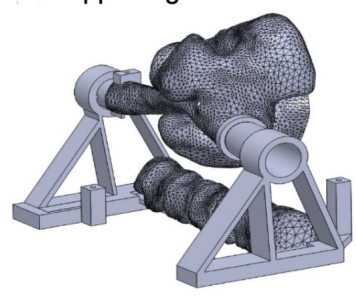

C Smoothing via Geomagic
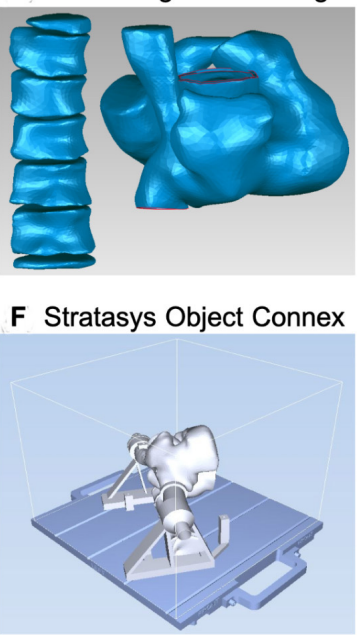

G Patient-specific 3D printed phantom

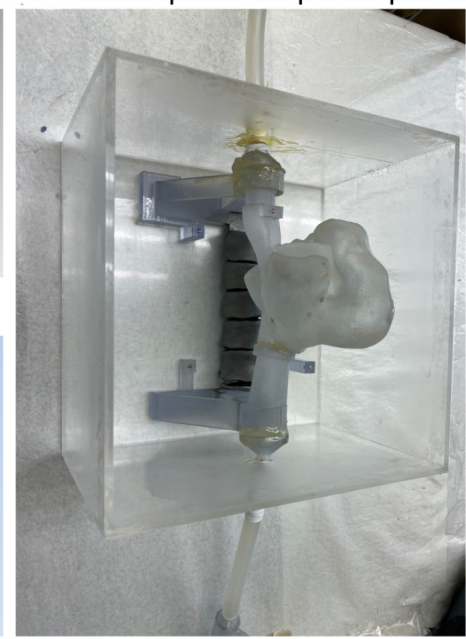

Figure 2. Depicting workflows of patient-specific 3D printed model. (A) Segmentation of heart and spine from DICOM file. (B, C) Import CAD into Geomagic Wrap for post-processing. (D, E) Import CAD into Solidworks to add support structures. (F, G) 3D print CAD, spray spine with metallized spray for opacity, and integrate both into acrylic box.

training set (60\%; 180 images); (2) validation set (20\%; 60 images); and (3) testing set (20\%; 60 images). The training and validation set were used during model training. The testing set was used for model evaluation at the end of the model training. To ensure that both our training and test dataset contain a fair representation of the catheter's tip and avoid overfitting, we randomly shuffled datasets before splitting them into training and test sets.

\section{Training}

The overall steps in our developments of a deep learning model are as follows: (1) randomly initialize the model; (2) train the model on the training set; (3) evaluate the trained model's performance on the validation set; (4) choose the model's hyperparameter with the best validation set performance; and (5) evaluate this chosen model on the test set. An adaptive moment (ADAM) estimation was used for training the $\mathrm{CNNs}^{[53]}$. The loss function was set to the binary cross-entropy. An early stopping rule was applied with 200 epochs. Finally, we evaluated the performance of the DL model by computing accuracy metrics and determined the Dice coefficient on the testing set. 


\section{Co-registration algorithms}

A key step in this system is to co-register the catheter and heart model in a single coordinate system. To this end, four metal spheres were embedded in our heart phantom model and used as fiducial markers. As shown in Figure 3A, the catheter and all four fiducial markers are visible in both of the bi-plane fluoroscopic images, such that they will be tracked and processed using the OpenCV library in Python. The OpenCV processing comprises Bitwise-Not operation, Smoothing operation, and Contours operation, illustrated in Figure $3 \mathrm{~B}$ and $\mathrm{C}$. Next, the radiopaque markers' $2 \mathrm{D}$ coordinates are identified from both fluoroscopic images $\left(\mathrm{RAO} 30^{\circ}, \mathrm{LAO}_{5} 5^{\circ}\right)$ and fed into the co-registration algorithms. Utilizing one of the radiopaque markers as a reference, the other coordinates will be offset. With the offset position of the fiducial marker and the known rotation angle, the $3 \mathrm{D}$ positions are solved from equation 3, as shown in Figure 3D. Then, the positions of four predefined fiduciary markers are used to calculate the affine transformation matrix in a single coordinate system using Eq. 4 and Eq. 5. The positions of four fiduciary markers are used to calculate the affine transformation matrix in a single coordinate system. Finally, the transformation matrix is applied to the position of the catheter's tip, as retrieved from a U-Net model prediction, to be co-registered in the coordinate system.

\section{RESULT AND DISCUSSION}

\section{Bi-plane co-registration accuracy}

To validate the accuracy of our $3 \mathrm{D}$ co-registration algorithm, we $3 \mathrm{D}$ printed a jig that holds an array of 50 metal spheres at various heights, shown in Figure 4. Using the biplane C-arm, two fluoroscopic images from two different angles were acquired and processed as described in section 2.5. Finally, the absolute error for each sphere was determined based on the difference between the true value measured from the 3D CAD file and the calculated value from the processed bi-plane images using our co-registration algorithm. As can be seen from Figure $4 \mathrm{C}$, the average accuracy was $0.12 \pm 0.11 \mathrm{~mm}$, which is highly accurate for cardiac interventions.

\section{Catheter tip detection}

The primary region of interest of a catheter during a procedure is its tip. Any intra-operative errors due to catheter tip maneuvering in the vascular system may raise the risk of puncture, embolization, or tissue damage ${ }^{[5,55]}$. As a result, we trained a deep learning U-Net model to detect the catheter tip's radiopaque marker in each frame of the fluoroscopic images. Figure 5 depicts the groundtruth and predicted segmentation of the catheter tip's radiopaque marker for the testing dataset. To evaluate the model performance, we used the area-based indexes to compare the predicted segmentation results with the groundtruth. These indexes include the Dice coefficient (DSC) $)^{[5]}$, Binary cross-entropy, and Intersection over Union (IOU) which can be found in Table 1. In order to improve the performance of the U-net model over our datasets and avoid the overfitting training phase, we performed extensive data augmentation ${ }^{[54]}$, including random shifting, scaling, rotation, and brightness/contrast changes, shown in Figure 6. Throughout each augmentation experiment, the IOU for each image and the mean average for the entire testing datasets (60 images) were calculated. We found that the best performance occurred by applying 10 random translations per image ( \pm 20 pixels), scaling with a zoom range of $0.1,10$ regular rotations per image, and random brightness and contrast of 0.5 resulting in $83.67 \%$ IOU. It should be noted that our reliable segmentation score (Dice of 0.8457 and IOU of 0.8367$)$ resulted in an accuracy of $(<1 \mathrm{~mm}$ ), which is far beyond the acceptable range for catheter tip tracking in cardiac applications.

To highlight the deep learning segmentation task's accuracy and efficiency, we compared the performance of the U-Net architecture with some classical image processing techniques (i.e., Thresholding, Watershed, Find and draw Contours by OpenCV, etc.). The catheter's radiopaque marker's appearance is affected by partial occlusions, intensity saturation, and motion blur. As can be seen from Figure 5, and despite the 
Table 1. Dice, Precision, and Recall metrics evaluation of catheter tip's radiopaque marker testing set for segmentation task by UNet model

\begin{tabular}{llll}
\hline Method & & \multicolumn{2}{l}{ Catheter tip's radiopaque marker segmentation } \\
\hline Indexes & Dice coefficient & Binary cross-entropy & Intersection over Union \\
\hline $\begin{array}{l}\text { Deep learning } \\
\text { U-Net model }\end{array}$ & 0.8457 & 0.3512 & 0.8367 \\
\hline
\end{tabular}
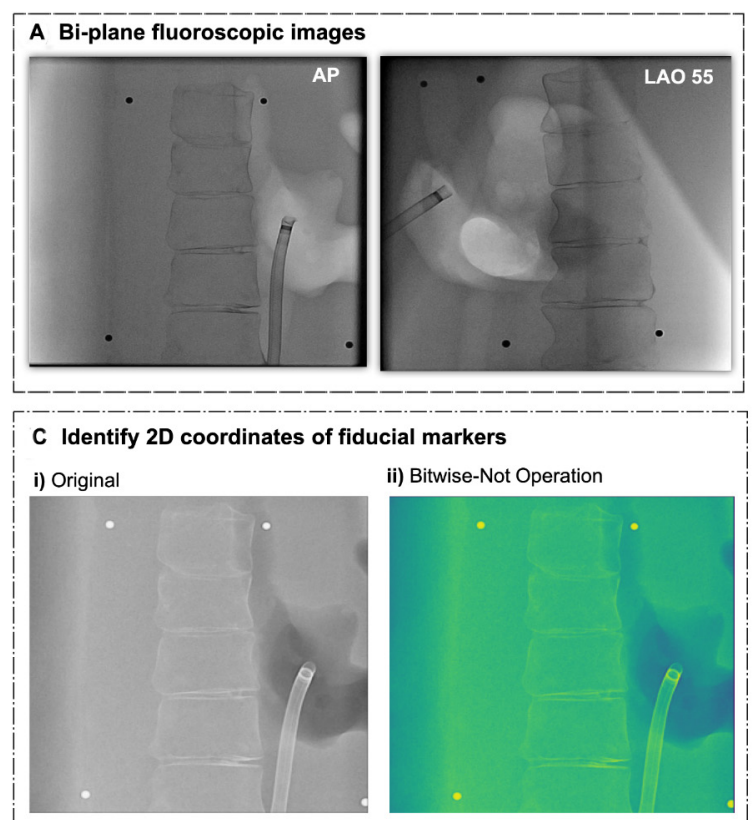

iii) Smoothing Operation

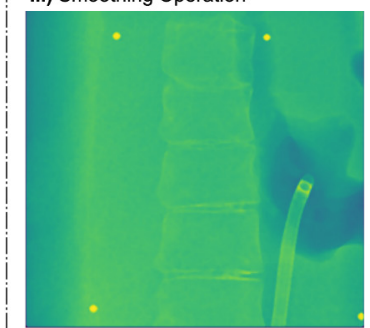

Bitwise-Not Operation

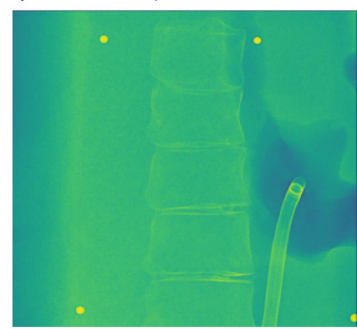

iv) Contours Operation

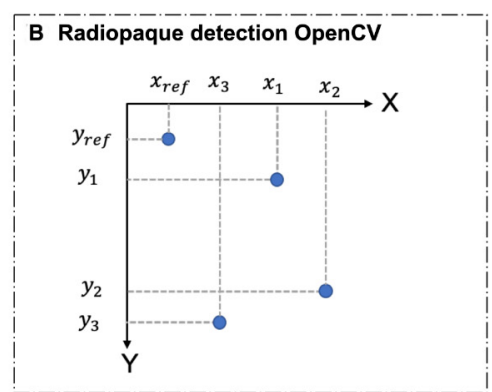

D Calculate transformation matrix

1.Off set

2. Scale

Angle No.1:

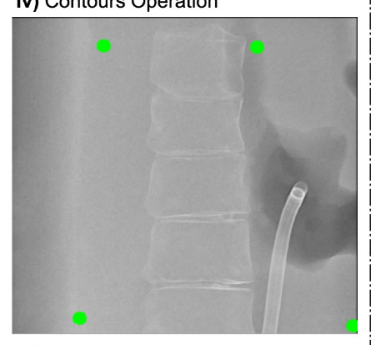

$X_{1}=\left[\begin{array}{lll}x_{11} & x_{12} & x_{13}\end{array}\right] \quad Y_{1}=\left[\begin{array}{lll}y_{11} & y_{12} & y_{13}\end{array}\right]$

Angle No.2:

$\mathrm{X}_{2}=\left[\begin{array}{lll}\mathrm{x}_{21} & \mathrm{x}_{22} & \mathrm{x}_{23}\end{array}\right] \quad \mathrm{Y}_{2}=\left[\begin{array}{lll}\mathrm{y}_{21} & \mathrm{y}_{22} & \mathrm{y}_{23}\end{array}\right]$

3. Coordinate geometry rotation

$\mathrm{Z}_{2}=\left(\mathrm{X}_{1}{ }^{*} \cos \theta-\mathrm{X}_{1}\right) / \sin \theta$

$\mathrm{Z}_{1}=\left(\mathrm{X}_{2}{ }^{*} \sin \theta\right)+\left(\mathrm{Z}_{2} * \cos \theta\right)$

4. Using angle No.1 and world coordinates

$A=\left[\begin{array}{lll}\mathrm{x}_{11} & \mathrm{x}_{12} & \mathrm{x}_{13} \\ \mathrm{y}_{11} & \mathrm{y}_{12} & \mathrm{y}_{13} \\ \mathrm{z}_{11} & \mathrm{z}_{12} & \mathrm{z}_{13}\end{array}\right]$

5. Calculate transformation matrix

$T=B * A^{-1}=B=\left[\begin{array}{lll}\mathrm{T}_{11} & \mathrm{~T}_{12} & \mathrm{~T}_{13} \\ \mathrm{~T}_{21} & \mathrm{y}_{22} & \mathrm{y}_{23} \\ \mathrm{~T}_{31} & \mathrm{~T}_{32} & \mathrm{~T}_{33}\end{array}\right]$

$T=B * A^{-1}=B=\left[\begin{array}{lll}\mathrm{T}_{21} & \mathrm{y}_{22} & \mathrm{y}_{23} \\ \mathrm{~T}_{31} & \mathrm{~T}_{32} & \mathrm{~T}_{33}\end{array}\right]$

6. Catheter tip 3D reconstruction

$M=\left[\begin{array}{lll}\mathrm{m}_{11} & \mathrm{~m}_{12} & \mathrm{~m}_{13}\end{array}\right] \& \quad P=T * M$

Figure 3. Illustration of sequential steps to co-register bi-plane fluoroscopic images (AP, LAO 55) utilizing four fiduciary radiopaque markers. (A) Raw fluoroscopic bi-plane images. (B) Radiopaque marker detection using OpenCV library. (C) Identify 2D coordinates of fiducial markers. (D) Co-registration algorithms to calculate the affine transformation matrix to combine all points into a single coordinate system.

widespread use of such methods (i.e., adaptive thresholding), they are prone to systemic noise and unreliable measurements, mainly due to the assumptions made in the computational design algorithms and failing to identify separable boundaries.

\section{Trajectory of catheter movement}

Fluoroscopy only provides a $2 \mathrm{D}$ projection image, and therefore no depth information is visible in the image ${ }^{[57]}$. Alternatively, fusion imaging allows for $3 \mathrm{D}$ imaging data of the heart tissue to be overlaid on a fluoroscopic image; but this technology has the drawback that the catheter and rendered tissue is only seen as a $2 \mathrm{D}$ projection, providing little to no post-procedural quantitative analysis. To this end, we demonstrate the $3 \mathrm{D}$ trajectory of the catheter derived from bi-plane co-registration method. The $3 \mathrm{D}$ trajectory of a 

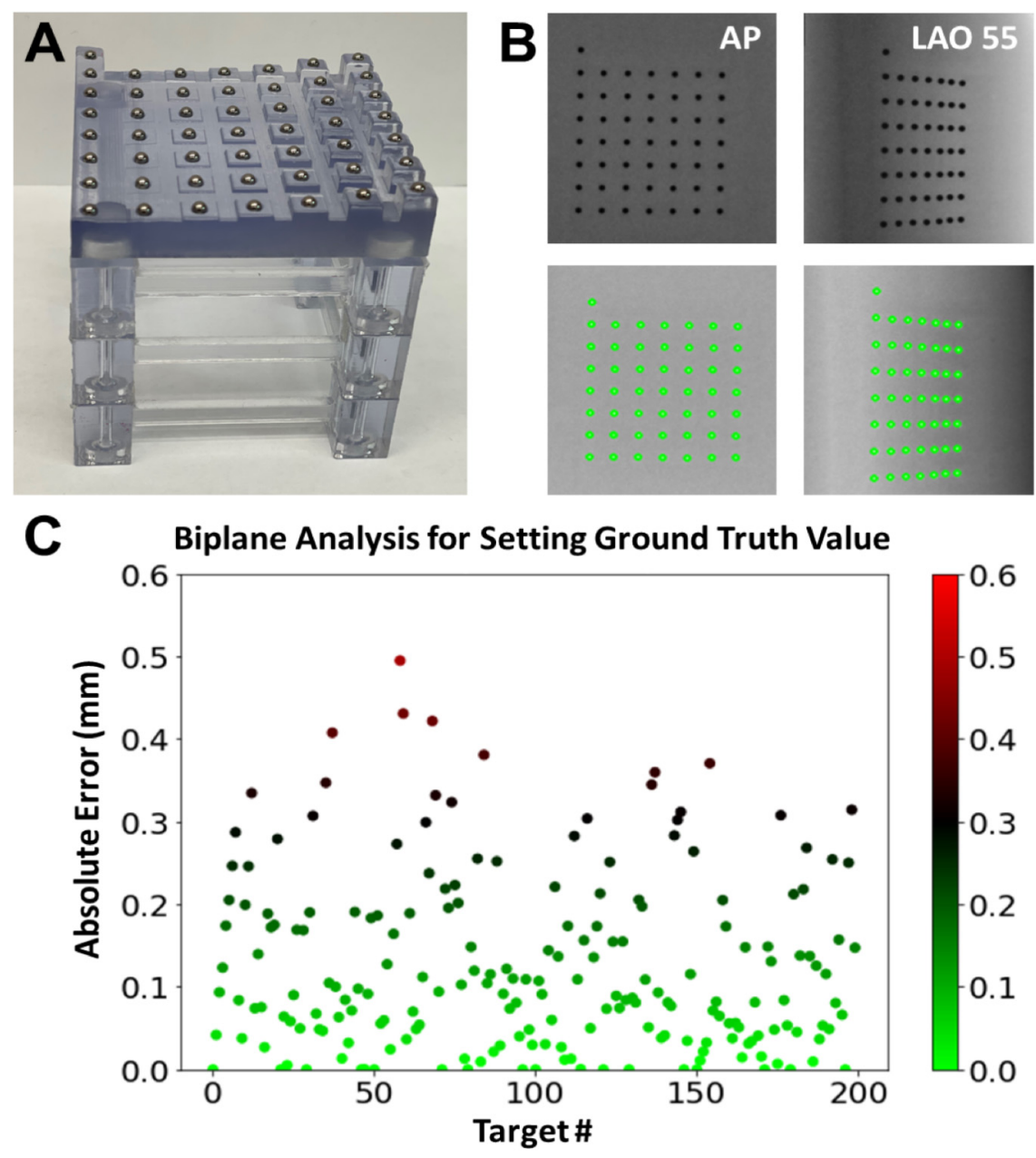

Figure 4. Validating 3D co-registration algorithm. (A) Image of 3D printed jig holding array of 50 metal spheres at various heights. (B) Image of fluoroscopy images at two angles and auto-detection of those spheres. (C) Graph of error for each sphere based on true value measured from 3D CAD file for bi-plane.

catheter is vital information for determining how a procedure was performed and providing a quantitative basis for analysis and future improvements. Figure 7A shows the selected fluoroscopic frames (LAO56 ${ }^{\circ}$, $\mathrm{RAO} 30^{\circ}$ ) acquired at the beginning and end of a mock procedure in the $3 \mathrm{D}$ printed model. After the catheter tip was detected from the two fluoroscopic images (i.e., $\mathrm{RAO} 30^{\circ}$, LAO56 $6^{\circ}$ ), the tip's coordinate (from $\mathrm{LAO} 56^{\circ}$ ) and the derived transformation matrix (from Eq. 5) was used to co-register the catheter in a single coordinate system as described earlier in section 2.5. Figure $7 \mathrm{~B}$ shows the catheter tip's $3 \mathrm{D}$ trajectory for the mock test.

\section{CONCLUSION}

This work demonstrates the implementation of a deep learning U-Net architecture to track the $3 \mathrm{D}$ movement of a catheter during a mock cardiac intervention under bi-plane fluoroscopy. We leveraged an end-diastolic cardiac CT in order to 3D print a patient-specific phantom model. We integrated four fiducial radiopaque markers on the phantom model, allowing us to co-register fluoroscopic images taken at two different angles (RAO30, LAO55). The U-Net model was trained in a supervised manner on the training set, and the trained model's performance was evaluated on the validation set. Finally, we assessed the DL model's performance by computing accuracy metrics and determining the Dice coefficient on the testing 

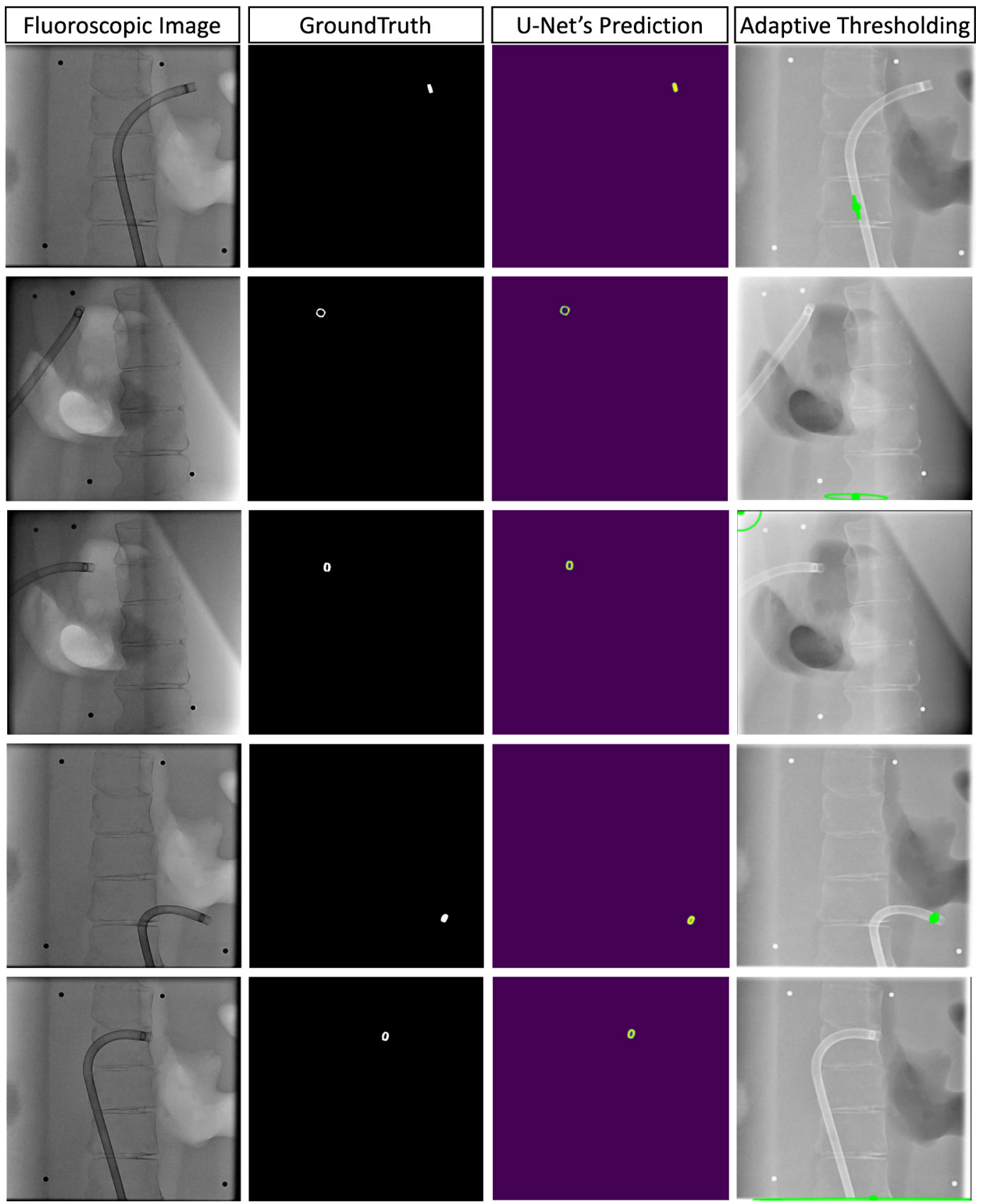

Figure 5. Comparison of catheter tip detection using the U-Net model and adaptive thresholding. Illustration of raw fluoroscopic images, groundtruth, and predicted segmentation for the two testing datasets.

set. Additionally, we demonstrated the 3D trajectory of the catheter tip's movement can be visualized graphically.

We believe the $3 \mathrm{D}$ trajectory analysis performed by this model can be used to analyze a physicians' performance and/or provide quantitative feedback for training and educational purposes. This work serves as a proof-of-principle that deep learning can be used for catheter tracking for cardiac interventions, however, since this article is a technical note, it has several limitations in its current stage, and we believe these limitations will be the seed for future developments for both our lab others. These limitations include: 


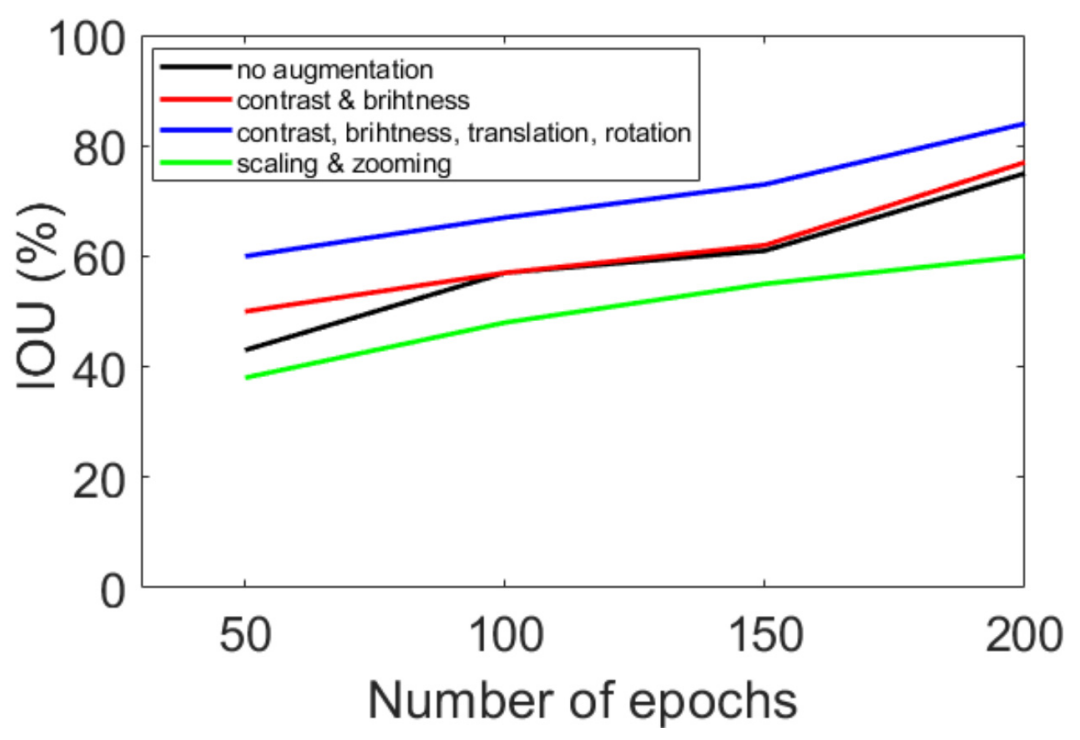

Figure 6. Relationship between the number of epochs for training, the data augmentation, and Intersection over Union (IOU). The applied augmentation including 10 regular rotations per image, 10 random translations per image $(+-20$ pixels $)$, brightness $=0.5$, contrast $=0.5$, scaling $=0.1$. The IOU percent is the mean average of IOU over 60 testing datasets.

A
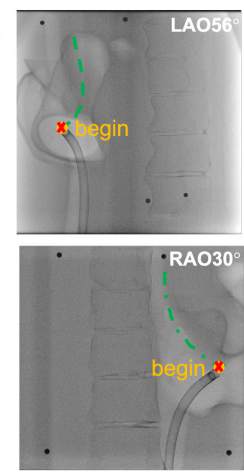
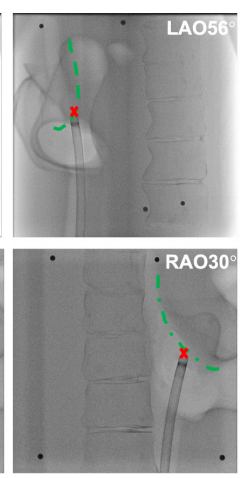
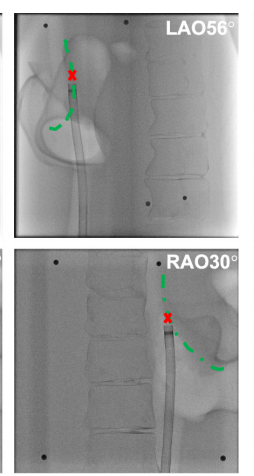

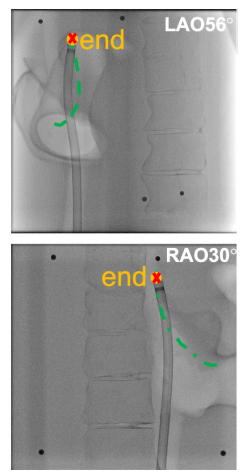

B 3D Trajectory of catheter tip - $\mathrm{LAO56}^{\circ}$

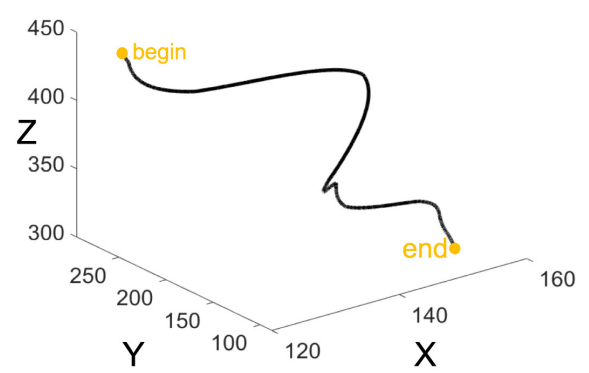

Figure 7. (A) Illustration of selected fluoroscopic frames ( $\mathrm{LAO} 56^{\circ}$ and $\mathrm{RAO} 30^{\circ}$ ) enclosing the beginning of mock procedures to the end. (B) $\mathrm{LAO56^{ \circ }} 3 \mathrm{D}$ trajectory of catheter tip retrieved from bi-plane co-registration.

(1) Limited data sets. Currently our dataset is only trained on a single 3D printed heart model and catheter. Therefore, a much more expansive dataset is needed to train a model that can accurately track catheters of different shapes and sizes and in hearts of differing anatomy. (2) Unrealistic background. Although these $3 \mathrm{D}$ printed models are patients-specific, meaning they accurately recapitulate the anatomy of the heart and spine, the fluoroscopic images don't include image artifacts from other surrounding anatomy, as will be the case for clinical images. (3) Limited analysis. Currently our model is only able to provide a 3D tracking of the catheter's tip, but there is no subsequent analysis to provide metrics for the performance of the intervention. This will require understanding the goals of the procedure and defining key metrics that can be quantified and will be useful for the physician. (4) No motion-compensation. The position of a catheter relative to the human heart is time-varying due to both respiration and cardiac contractions. Since we're using a $3 \mathrm{D}$ printed model there was no motion to compensate for, however, solutions will need to be integrated for the catheter tracking to properly co-register the catheter tip to the heart in a clinical procedure. (5) Spherical fiducial markers. Since a 3D printed model was used, it was convenient to use metal spheres as extrinsic fiducial markers. However, placement of these spheres on an individual will not 
be trivial and therefore methods that utilize the spine as an intrinsic fiducial maker should be used during acquisition of clinical images, as described in our previous work ${ }^{[58]}$.

Due to the above listed limitations, this work will have the most immediate impact for performing quantitative analysis of training procedure on $3 \mathrm{D}$ printed heart models. We expect that more sophisticated heart models that include motion and match disease states will be created, along with specific criteria for success for each model/intervention to provide feedback in the form of quantitative metrics. Furthermore, the ability to process images in real-time and display the catheter in MR renderings will improve training by providing assistance during the training session, as described in our previous work that adopted EM sensors for tracking ${ }^{[35]}$. We believe this tracking system will serve to lower the learning curve for new fellows and refine the procedural techniques of attendings.

\section{DECLARATIONS}

\section{Acknowledgments}

We thank the Dalio Institute of Cardiovascular Imaging for their support and funding.

\section{Authors' contributions}

Conceived of the presented work: Mosadegh B, Torabinia M

Took the lead in writing the manuscript: Torabinia M, with support from Mosadegh B

Carried out the experiment: Caprio A, Torabinia M

Involved in processing and analyzing the datasets: Jang S, Ma T, Tran H, Mekki L, Chen I, led by Torabinia $\mathrm{M}$

Supervised the theoretical and deep learning framework: Sabuncu M, Mosadegh B

Supervised the image acquisition: Wong S, Mosadegh B

All authors discussed the results and commented on the manuscript.

\section{Availability of data and materials}

Not applicable.

\section{Conflicts of interest}

All authors declared that there are no conflicts of interest.

\section{Ethical approval and consent to participate}

Not applicable.

\section{Consent for publication}

Not applicable.

\section{Copyright}

(c) The Author(s) 2021.

\section{REFERENCES}

1. Niu G, Pan B, Zhang F, Feng H, Fu Y. Improved surgical instruments without coupled motion used in minimally invasive surgery. Int J Med Robot 2018;14:e1942. DOI PubMed

2. Bello B, Herbella FA, Allaix ME, Patti MG. Impact of minimally invasive surgery on the treatment of benign esophageal disorders. World J Gastroenterol 2012;18:6764-70. DOI PubMed PMC

3. Gjeraa K, Spanager L, Konge L, Petersen RH, Østergaard D. Non-technical skills in minimally invasive surgery teams: a systematic review. Surg Endosc 2016;30:5185-99. DOI PubMed

4. Eswara JR, Ko DS. Minimally invasive techniques in urology. Surg Oncol Clin N Am 2019;28:327-32. DOI PubMed

5. Schatz C. Enhanced recovery in a minimally invasive thoracic surgery program. AORN J 2015;102:482-92. DOI PubMed

6. Devgan L, Singh P, Durairaj K. Minimally invasive facial cosmetic procedures. Otolaryngol Clin North Am 2019;52:443-59. DOI 
PubMed

7. Mack MJ. Minimally invasive cardiac surgery. Surg Endosc 2006;20 Suppl 2:S488-92. DOI PubMed

8. Gillion JF, Fagniez PL. Chronic pain and cutaneous sensory changes after inguinal hernia repair: comparison between open and laparoscopic techniques. Hernia 1999;3:75-80. DOI

9. Dedemadi G, Sgourakis G, Karaliotas C, Christofides T, Kouraklis G, Karaliotas C. Comparison of laparoscopic and open tension-free repair of recurrent inguinal hernias: a prospective randomized study. Surg Endosc 2006;20:1099-104. DOI PubMed

10. Subramanian VA, Mccabe JC, Geller CM. Minimally invasive direct coronary artery bypass grafting: two-year clinical experience. Ann Thorac Surg 1997;64:1648-55. DOI PubMed

11. Stevens JH, Burdon TA, Peters WS, et al. Port-access coronary artery bypass grafting: a proposed surgical method. $J$ Thorac Cardiovasc Surg 1996;111:567-73. DOI PubMed

12. Ota T, Degani A, Schwartzman D, et al. A highly articulated robotic surgical system for minimally invasive surgery. Ann Thorac Surg 2009;87:1253-6. DOI PubMed PMC

13. Pyciński B, Juszczyk J, Bożek P, Ciekalski J, Dzielicki J, Pietka E. Image navigation in minimally invasive surgery. In: Piętka E, Kawa J, Wieclawek W, editors. Information technologies in biomedicine, volume 4. Cham: Springer International Publishing; 2014. p. 25-34.

14. Antico M, Sasazawa F, Wu L, et al. Ultrasound guidance in minimally invasive robotic procedures. Med Image Anal 2019;54:149-67. DOI PubMed

15. Pisano GP, Bohmer RM, Edmondson AC. Organizational differences in rates of learning: evidence from the adoption of minimally invasive cardiac surgery. Management Science 2001;47:752-68. DOI

16. Moorthy K, Munz Y, Dosis A, et al. Dexterity enhancement with robotic surgery. Surg Endosc 2004;18:790-5. DOI PubMed

17. Milano EG, Capelli C, Wray J, et al. Current and future applications of 3D printing in congenital cardiology and cardiac surgery. $\mathrm{Br} J$ Radiol 2019;92:20180389. DOI PubMed PMC

18. Min JK, Mosadegh B, Dunham S, Al'Aref SJ. 3D Printing applications in cardiovascular medicine. Cambridge: Academic Press; 2018.

19. Vukicevic M, Mosadegh B, Min JK, Little SH. Cardiac 3D printing and its future directions. JACC Cardiovasc Imaging 2017;10:17184. DOI PubMed PMC

20. Biglino G, Capelli C, Binazzi A, et al. Virtual and real bench testing of a new percutaneous valve device: a case study. EuroIntervention 2012;8:120-8. DOI PubMed

21. Schmauss D, Haeberle S, Hagl C, Sodian R. Three-dimensional printing in cardiac surgery and interventional cardiology: a singlecentre experience. Eur J Cardiothorac Surg 2015;47:1044-52. DOI PubMed

22. Ryan JR, Moe TG, Richardson R, Frakes DH, Nigro JJ, Pophal S. A novel approach to neonatal management of tetralogy of Fallot, with pulmonary atresia, and multiple aortopulmonary collaterals. JACC Cardiovasc Imaging 2015;8:103-4. DOI PubMed

23. Chaowu Y, Hua L, Xin S. Three-dimensional printing as an aid in transcatheter closure of secundum atrial septal defect with rim deficiency: in vitro trial occlusion based on a personalized heart model. Circulation 2016;133:e608-10. DOI PubMed

24. Sodian R, Weber S, Markert M, et al. Stereolithographic models for surgical planning in congenital heart surgery. Ann Thorac Surg 2007;83:1854-7. DOI PubMed

25. Noecker AM, Chen JF, Zhou Q, et al. Development of patient-specific three-dimensional pediatric cardiac models. ASAIO J 2006;52:349-53. DOI PubMed

26. Vranicar M, Gregory W, Douglas WI, Di Sessa P, Di Sessa TG. The use of stereolithographic hand held models for evaluation of congenital anomalies of the great arteries. Stud Health Technol Inform 2008;132:538-43. PubMed

27. Schievano S, Migliavacca F, Coats L, et al. Percutaneous pulmonary valve implantation based on rapid prototyping of right ventricular outflow tract and pulmonary trunk from MR data. Radiology 2007;242:490-7. DOI PubMed

28. Vukicevic M, Conover T, Jaeggli M, et al. Control of respiration-driven retrograde flow in the subdiaphragmatic venous return of the Fontan circulation. ASAIO J 2014;60:391-9. DOI PubMed PMC

29. Garekar S, Bharati A, Chokhandre M, et al. Clinical application and multidisciplinary assessment of three dimensional printing in double outlet right ventricle with remote ventricular septal defect. World J Pediatr Congenit Heart Surg 2016;7:344-50. DOI PubMed

30. Deferm S, Meyns B, Vlasselaers D, Budts W. 3D-printing in congenital cardiology: from flatland to spaceland. J Clin Imaging Sci 2016;6:8. DOI PubMed PMC

31. Kiraly L, Tofeig M, Jha NK, Talo H. Three-dimensional printed prototypes refine the anatomy of post-modified Norwood-1 complex aortic arch obstruction and allow presurgical simulation of the repair. Interact Cardiovasc Thorac Surg 2016;22:238-40. DOI PubMed

32. Biglino G, Capelli C, Taylor AM, Schievano S. 3D Printing Cardiovascular Anatomy: A Single-Centre Experience. In: Shishkovsky IV, editor. New Trends in 3D Printing. IntechOpen; 2016. DOI

33. Anwar S, Singh GK, Varughese J, et al. 3D printing in complex congenital heart disease: across a spectrum of age, pathology, and imaging techniques. JACC Cardiovasc Imaging 2017;10:953-6. DOI PubMed

34. Olivieri LJ, Krieger A, Loke YH, Nath DS, Kim PC, Sable CA. Three-dimensional printing of intracardiac defects from threedimensional echocardiographic images: feasibility and relative accuracy. J Am Soc Echocardiogr 2015;28:392-7. DOI PubMed

35. Jang S, Torabinia M, Dhrif H, et al. Development of a hybrid training simulator for structural heart disease interventions. Advanced Intelligent Systems 2020;2:2000109. DOI

36. Girshick R, Donahue J, Darrell T, Malik J. Rich feature hierarchies for accurate object detection and semantic segmentation. 2014 IEEE Conference on Computer Vision and Pattern Recognition; 2014 Jun 23-28; Columbus, USA. 2014.

37. Jia Y, Shelhamer E, Donahue J, et al. 2014. Caffe: convolutional architecture for fast feature embedding. In Proceedings of the 22nd 
ACM international conference on Multimedia (MM '14); New York, USA. 2014.

38. Sermanet P, Eigen D, Zhang X, Mathieu M, Fergus R, LeCun Y. Overfeat: Integrated recognition, localization and detection using convolutional networks. 2013

39. Chandan G, Jain A, Jain H, Mohana. Real time object detection and tracking using deep learning and OpenCV. 2018 International Conference on Inventive Research in Computing Applications (ICIRCA); 2018 Jul 11-12; Coimbatore, India. 2018.

40. Rajchl M, Lee MC, Oktay O, et al. DeepCut: object segmentation from bounding box annotations using convolutional neural networks. IEEE Trans Med Imaging 2017;36:674-83. DOI PubMed PMC

41. Ronneberger O, Fischer P, Brox T. U-Net: convolutional networks for biomedical image segmentation. In: Navab N, Hornegger J, Wells W, Frangi A, editors. Medical image computing and computer-assisted intervention - MICCAI 2015. Cham: Springer; 2015. p. 234-41.

42. Shelhamer E, Long J, Darrell T. Fully convolutional networks for semantic segmentation. IEEE Trans Pattern Anal Mach Intell 2017;39:640-51. DOI PubMed

43. Çiçek Ö, Abdulkadir A, Lienkamp SS, Brox T, Ronneberger O. 3D U-Net: learning dense volumetric segmentation from sparse annotation. In: Ourselin S, Joskowicz L, Sabuncu M, Unal G, Wells W, editors. Medical image computing and computer-assisted intervention - MICCAI 2016. Cham: Springer; 2016. p. 424-32.

44. Milletari F, Navab N, Ahmadi S. V-Net: fully convolutional neural networks for volumetric medical image segmentation. 2016 Fourth International Conference on 3D Vision (3DV); 2016 Oct 25-28; Stanford, USA. 2016.

45. Li X, Chen H, Qi X, Dou Q, Fu CW, Heng PA. H-DenseUNet: hybrid densely connected UNet for liver and tumor segmentation from CT volumes. IEEE Trans Med Imaging 2018;37:2663-74. DOI PubMed

46. Zhou Z, Rahman Siddiquee MM, Tajbakhsh N, Liang J. UNet++: a nested U-Net architecture for medical image segmentation. In: Stoyanov D, Taylor Z, Carneiro G, Syeda-mahmood T, Martel A, Maier-hein L, Tavares JMR, Bradley A, Papa JP, Belagiannis V, Nascimento JC, Lu Z, Conjeti S, Moradi M, Greenspan H, Madabhushi A, editors. Deep learning in medical image analysis and multimodal learning for clinical decision support. Cham: Springer International Publishing; 2018. p. 3-11.

47. Zhang J, Jin Y, Xu J, Xu X, Zhang Y. Mdu-net: multi-scale densely connected u-net for biomedical image segmentation. 2018.

48. Jin Q, Meng Z, Pham TD, et al. DUNet: a deformable network for retinal vessel segmentation. Knowledge-Based Systems 2019;178:149-62. DOI

49. Jin Q, Meng Z, Sun C, Cui H, Su R. RA-UNet: a hybrid deep attention-aware network to extract liver and tumor in CT scans. Front Bioeng Biotechnol 2020;8:605132. DOI PubMed PMC

50. Dolz J, Ben Ayed I, Desrosiers C. Dense multi-path U-Net for ischemic stroke lesion segmentation in multiple image modalities. In: Crimi A, Bakas S, Kuijf H, Keyvan F, Reyes M, van Walsum T, editors. Brainlesion: glioma, multiple sclerosis, stroke and traumatic brain injuries. Cham: Springer; 2018. p. 271-82.

51. Xiao W, Duan X, Lin Y, et al. Distinct proteome remodeling of industrial saccharomyces cerevisiae in response to prolonged thermal stress or transient heat shock. J Proteome Res 2018;17:1812-25. DOI PubMed

52. Isensee F, Petersen J, Klein A, et al. nnu-net: self-adapting framework for u-net-based medical image segmentation. 2018.

53. Kingma DP, Ba J. Adam: a method for stochastic optimization. Computer Science 2014.

54. Fukumoto Y, Tsutsui H, Tsuchihashi M, Masumoto A, Takeshita A. The incidence and risk factors of cholesterol embolization syndrome, a complication of cardiac catheterization: a prospective study. J Am Coll Cardiol 2003;42:211-6. DOI PubMed

55. Loffroy R, Guiu B, Cercueil JP, Krausé D. Endovascular therapeutic embolisation: an overview of occluding agents and their effects on embolised tissues. Curr Vasc Pharmacol 2009;7:250-63. DOI PubMed

56. Dice LR. Measures of the amount of ecologic association between species. Ecology 1945;26:297-302. DOI

57. Sra J, Krum D, Choudhuri I, et al. Identifying the third dimension in $2 \mathrm{D}$ fluoroscopy to create $3 \mathrm{D}$ cardiac maps. JCI Insight 2016;1:e90453. DOI PubMed PMC

58. Liu J, Al'Aref SJ, Singh G, et al. An augmented reality system for image guidance of transcatheter procedures for structural heart disease. PLoS One 2019;14:e0219174. DOI PubMed PMC 